\title{
Sensing the epilepsy brain: a unique nanosurgical biomedical device to treat the epilepsy patient
}

\begin{abstract}
A sleek nanobiosensor, BRODERICK PROBE ${ }^{\circledR}$, smaller than a human hair, is designed to diagnose and treat debilitating neurodegenerative and psychiatric brain disorders. This editorial focuses on this unique series of nanobiosensors specifically as a miniature nanosurgical biomedical device for the epilepsy patient and for the first time, the epilepsy brain is sensed, that is, these nanobiosensors sense or see inside the brain of the epilepsy patient, actually intra-operatively. These sensors perform live imaging during resection surgery needed to treat the epilepsy patient who presents with epilepsy seizures intractable to pharmacotherapeutics, that is, those patients who are unable to medically respond to the pharmaceutical regimen often used effectively in the course of epilepsy patient treatment.

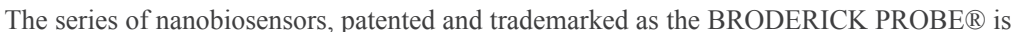
comprised of biologically compatible materials, capable of imaging neurotransmitter signals directly from the brain, in fact, during movement, during the natural state, the diseased state and the medically/surgically treated state in the same subject in real time. It is the first series of nanobiosensors in the world that is capable of, video tracking "visualizing" brain neurotransmitters as signals for each neurotransmitter are seen in real time on a computer/ mobile device. This series of nanobiosensors is minimally invasive using one method ${ }^{1-12}$ as well as non-invasive using another method., ${ }^{1,2,3}$ The nanobiosensors, collectively trademarked BRODERICK PROBE $®$ image the brain, opens the brain without actually opening the brain and does not harm the brain. Controlled studies have shown that neither, the nanobiosensor, per se, its reference or counter, nor its auxiliary component enables bacterial infection in long term studies according to NYU Immunology Tierno team. This nanobiosensor has proven clinically to be successful in the epilepsy patient without producing scar tissue or gliosis, as studies by NYU pathologist.Glial Fibrillary Acidic Protein (GFAP) stains of tissue were post-surgically conducted in order to assess gliosis or other neuronal damage after nanobiosensor, reference/counter or auxiliary placement. Therefore, based on the pathology report from the NYU surgical team, the BRODERICK $\mathrm{PROBE}^{\circledR}$ nanobiosensors did not cause gliosis, sclerosis, brain/neuronal damage, or histiocyte infiltration and are, thus, a safe biomedical tool.

Therefore, sensing nanotechnology, the science of advanced materials, is introduced into the entire field of neurodegenerative and psychiatric brain disorders as proven in mammalian animals and humans and the success has extrapolated from laboratory to clinic in debilitating seizure disorders as demonstrated in its effective and safe Imaging in patients with epilepsy intraoperatively. It is noteworthy that this sleek nanobiosensor enables direct imaging, a live imaging, a look, inside the brain. This live imaging is coined Neuromolecular Imaging, abbreviated as NMI.
\end{abstract}

Volume 8 Issue 2 - 2018

\section{Patricia Ann Broderick \\ Department of Molecular, Cellular, and Biomedical Sciences, City} University of New York (CUNY), USA

Correspondence: Patricia Ann Broderick, Department of Molecular, Cellular, and Biomedical Sciences, City University of New York (CUNY), USA, Tel +7l 8-928-4858,

Email drpabroderick@gmail.com

Received: March 18, 2018 | Published: March 23, 2018

\section{BRODERICK PROBE ${ }^{\circledR}$ nanobiosensors}

The BRODERICK PROBE ${ }^{\circledR}$ encompasses several formulations of carbon-based biosensors, patented by City University of New York (CUNY) and assigned to CUNY and in part to NYU Medical Center of NYU; all patents describing in detail, the series of nanobiosensors are held by Eazysense Nanotechnologies Incorporated in New York State in concert with CUNY Technology Commercialization Office (TCO). Nanobiosensors were tested in controlled research paradigms..$^{1,5,11,13-17}$ A description of specific components for each nanobiosensor formulation, as well as the use, design and applications for these nanobiosensors, are published; detector/potentiostat electrical circuits and associated NMI biotechnology are also published in depth. . $, 3,6-10,12^{-12}$

Epilepsy is a disease of the central nervous system wherein a bundle of neurons suddenly go awry and excessive firing of several neuronal cells release neurochemicals into the synapse, and cause dramatically differential subtypes of motor, emotional, cognitive and mental disorders in addition to trauma and death in neuronal issues. Epilepsy involves seizures where the brain is causally involved in the neuropathy; nonetheless, it is noteworthy that not all seizures are, in truth, epilepsy. The neuroanatomic sites where epileptic seizures occur are, amazingly, in each and every lobe of the brain, temporal, frontal, parietal and occipital, However, temporal lobe surgery, in particular, is most amenable to success for resection surgery. Simply stated, these intensely excessive neuronal depolarization (firing) of seizing neurons can cause generalized or partial (focal) seizures, wherein the latter type diagnoses can be confused with a diagnoses of meningitis, that is inflamed meningeal films that cover the brain under the skull.

Since the 1990's, pharmacotherapeutics for epilepsy patients has progressed in geometric proportion. Still, patients remain intractable, unable to respond to these pharmaceutics to stop epilepsy seizures and attempt to achieve quality of life. Hence, surgery has come into greater significance in terms of recovery from epilepsy seizures. As mentioned earlier, temporal lobe epilepsy patients, either neocortical 
and/or mesial, show approximately 90 percent successful outcome for patients undergoing surgical procedures such as the conventional resection surgery, albeit the epileptic zone range for abnormal neurons remains elusive. Here, we present a unique nanosurgical medical device to treat the epilepsy patient and moreover, use a sensing device to image neurotransmitters in the epilepsy brain online and in Vivo, Thus, the BRODERICK PROBE $®$ (named after the author's father) is the first to introduce sensing medical devices into the field of epilepsy therapy and especially, into the surgical treatment of epilepsy patients.

In depth, an advanced biomedical device for the diagnosis of epilepsy in patients during surgery is described. This micronanotechnologic device represents a significant advance in biomedical engineering and bioimaging. The modus operandi underlying normal and pathologic neuronal function in the intact brain of the epilepsy patient is defined, in Vivo, using nanobiosensors patented by CUNY and in part by NYU, approved by the Internal Review Board at NYU. Using novel semiderivative voltammetric electrical circuits, patented by Broderick at CUNY, Neuromolecular Imaging (NMI), with the BRODERICK PROBE ${ }^{\circledR}$ nanobiosensor, the selective detection of specific neurotransmitters in discrete parts of intact brain are imaged within a temporal resolution of seconds and subseconds. Presurgical evaluation of the patient with electroencephalographic (EEG) monitoring identified the site for neocortical resection; subdural grid and strip electrodes, placed on the brain's surface sought the focus of specific epileptogenic cortex.

Nanobiosensors that actually have a diameter five times less than invasive EEG epilepsy depth electrodes directly visualized and video tracked neurochemical profile on the site of the exposed cortical region and did so with and without epileptic spike activity in regions destined for resection. About six to ten recordings with $\gamma$-irradiated (11.6-12.7 kGy), laminar biocompatible carbon-based laurate nanobiosensors were taken at a cortical depth of 2 to $4 \mathrm{~mm}$ for a 20-30 min time period. Biomarkers were imaged with signals such as excitatory dynorphin and neuroprotective somatostatin. Intraoperative images were verified by comparison with our more than fifteen year empirical database from epilepsypatients. ${ }^{6,7,9,10,12}$

Figure 3 during resection surgery in the epilepsy patient, the BRODERICK PROBE ${ }^{\circledR}$ nanobiosensor, smaller than one human hair, is placed, intra operatively, directly on the exposed brain of the epilepsy patient in the anterior temporal lobe of patients' brain. Readily seen is what is called, the "Great Vein".

This series of nanobiosensors is minimally invasive using one method $^{4,6,7,9,12}$ as well as non-invasive using another method. ${ }^{2,3,8}$ In the first part, the nanobiosensor is carbon based and in the second part, the nanobiosensor is protein based.

In the first part, this novel nanotechnology that senses the brain is comprised of the potentiostat detector, the nanobiosensor input and electrochemical detection as seen online as computer image, described in Figure 1 and Figure 2. In the second part, the novel nanotechnology that senses the brain is comprised of a dual photo-laser diode protein based nanobiosensor input and fiber optics input for electrochemical spectroscopic output detection. Figure 4 shows a schematic of a generic photodiode. Figure 2 shows the live resected hippocampus surgically removed after study. The live temporal lobe in the epilepsy brain is shown, exposed, intraoperatively in Figure 3. A schematic diagram of the generic BRODERICK PROBE ${ }^{\circledR}$ is shown in Figure 1.

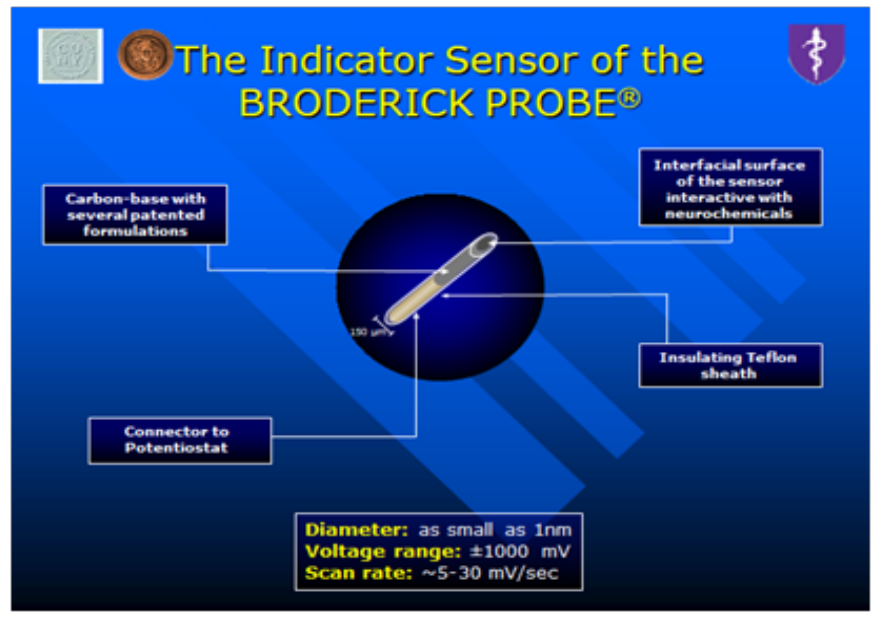

Figure 1 Schematic drawing of just one of several designs for the BRODERICK $\mathrm{PROBE}^{\circledR}$ series of nanobiosensors.

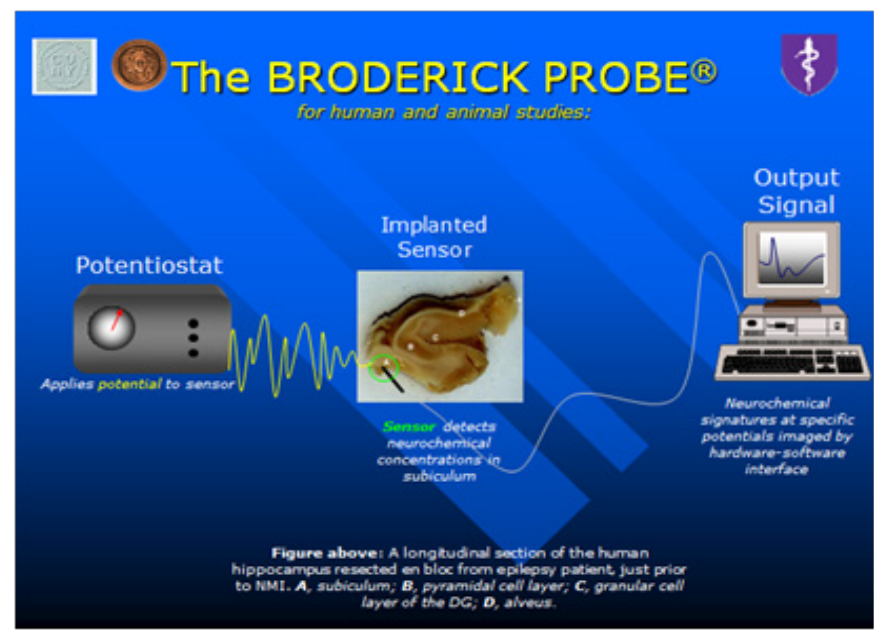

Figure 2 A longitudinal section of the human hippocampus resected en bloc from epilepsy patient, just prior to NMI. A: subiculum; B: pyramidal cell layer; C: granular cell layer of the dentate gyrus (DG); D: alveus.

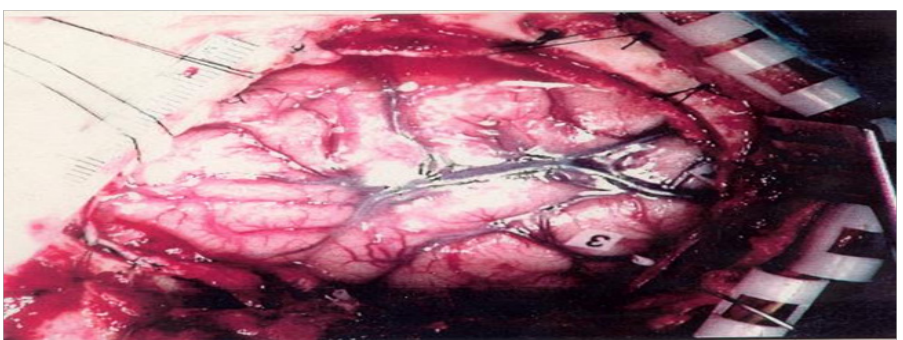

Figure 3 The exposed brain is which the BRODERICK PROBE ${ }^{\circledR}$ nanobiosensor is placed. 


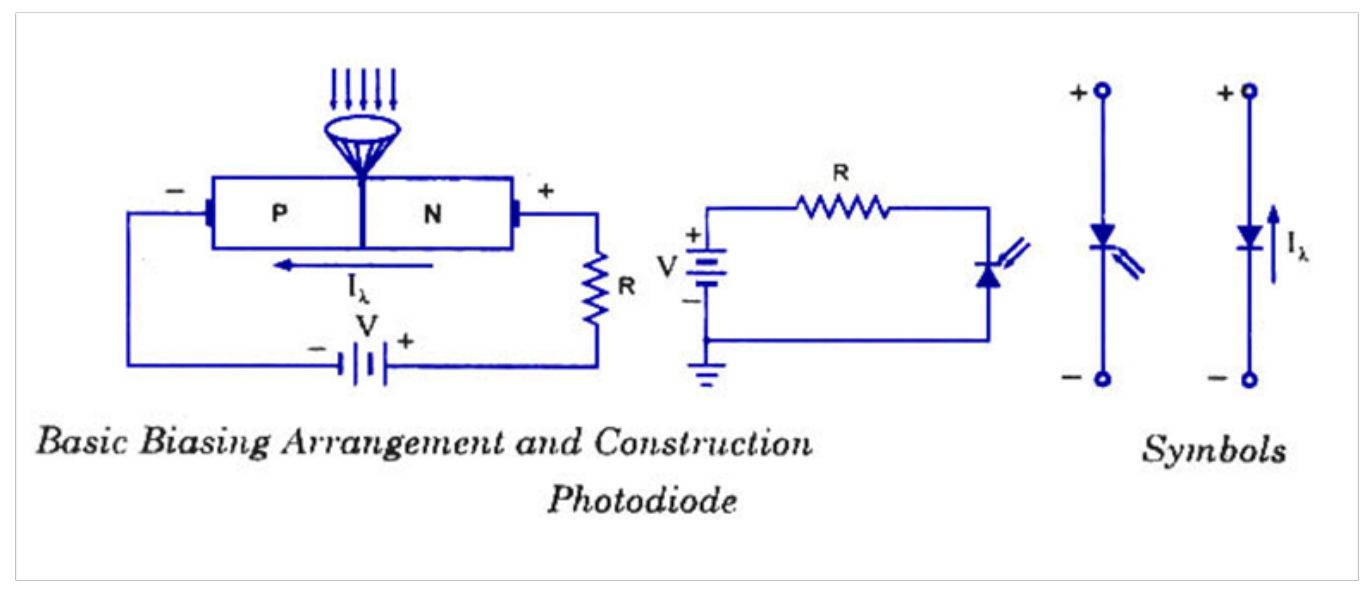

Figure 4 shows a basic biasing arrangement and construction of a photodiode in schematic, generic model.

Nanotechnology: sensing the brain: the journey from in vivo electrochemistry to neuromolecular imaging to voltaic photonics

\section{Quantum Mechanics:The newest of the new inventive sensing art}

What is quantum mechanics? In short form,one may say, just pass a voltage across a household laser emitted diode (LED) designed to read in the visible spectral analysis range and an electron is released!

Thus, the newest of the new art, the BRODERICK PROBE® protein neuroprobe is comprised of a series of photosensitive, electroactive, polymer sensing devices or nanosensors that operates by converting photonic energy into electrochemical energy to image neurochemicals and neurotransmitters in real time; it is non-invasive and is well suited to live image neurotransmitter profiles in neurodegenerative and psychiatric disorders in humans and animals such as diseases of the motor and sensory systems, for example, the eye, brain, and skin. The sensing device or nanosensor or neuroprobe is used to treat patients and animals with brain diseases. The sensing device enables the inside of the brain to be video tracked without opening the brain, as is conventionally required. In doing so, this new art provides substantial advancements in personalized medicine for diagnosis and treatment in, for example, Parkinson's with Lewy Body Alzheimer's disease, epilepsy, stroke, and other neurodegenerative and psychiatric brain diseases that occur and traumatize brain and induce movement and cognitive decline in the process. This newest of the new art enables live imaging of neurotransmitters and by implication, genes associated with each neurotransmitter and neurochemical.

\section{How does it work?}

Photons of light carrying proteins, such as opsins through ligand gated channels in the skull and brain. An electrochemical current, based on the photonic light energy, enables the conversion of light energy to electrical energy and creates a photoelectrical current in units and subunits of voltage. This photoelectrochemical current is monitored using a potentiostat, spectrometer, and/or a spectroelectrochemical chemiluminesence device. What we have here is a miniature photosensitive, electroactive polymer sensing device or nanosensor or neuroprobe that operates by converting photonic energy into electrical and electrochemical energy that generates a photocurrent in the brain without opening the brain and/ or opening the brain minimally. The output is provided in units of voltage. Laser diodes then enable the electrochemical waveform to be seen as an electrochemical image in color separated into specific spectral frequencies. Thus, the photocurrent provides an image of neurochemicals in real time, noninvasively and/or minimally invasively. Thus, enabled by the quantum mechanical world of advanced molecular science and medicine as well as nanodevices, the BRODERICK PROBE $®$ photoelectrochemical conductance device provides another novel series of nanosensors for nanodiagnostics, nanotherapies, and nanotheranostics.

These quantum mechanical devices, based on the protein probe by Broderick, includes a memory transduction device enabled by polymeric molecular bonding that utilizes highly polar covalent hydrogen bonding intermolecularly. The art includes electrical devices that enable the discovery of previously "difficult to reach" target compounds. The art enables diagnoses and treatment of disease via the use of anion-cation exchange properties within electrochemical reduction voltammetry control.

In fact, the fiber optic, voltaic neuroprobe utilizes additional proteins that are luminescent. Human LIGHT is a type II membrane protein that is a member of the TNF superfamily (TNFSF14). LIGHT is an acronym for Lymphotoxins exhibit Inducible expression, and competes with HSV Glycoprotein D for HVEM, a receptor expressed by T-lymphocytes. LIGHT is also called HVEM-L and LT-gamma photosensitive serine protease inhibitors. The art relates to inhibitors for serine proteases (thrombin, factor $\mathrm{Xa}$, and chymotrypsin, important in blood clotting mechanisms and hence, stroke) that are light sensitive. In addition, this exciting art also include a protein or protein induced photosensitive material made by using hydrophobic bonds from phosphatidylcholine incorporated into the protein, such as we have patented and accomplished with lipid and carbon allotropes $6,7,9,10,12$ and also by using hydrophobic bonds of the photolysed complex with protease from staphylococcus aureus and trypsin.

The protein, albumin, can also be made photosensitive. Hence, further, a method of making proteins luminescent and making 
other materials that are not proteins, luminescent, is added to the armamentarium. The new devices include chemiluminescent materials that are or may not even be photonic, but emit the advanced materials made in our laboratory will enable light to emit during catalysis when a second compound is catalysed by another compound producing a different compound by reassembly of electrons by catalytic amplification. Other means of amplification can likely occur by lipid in addition to protein-lipid amplification. ${ }^{2,3,8}$

Figure 4 shows a basic biasing arrangement and construction of a photodiode in schematic, generic model form emphasizing the semiconductor properties of a luminescent photodiode at the $\mathrm{P} / \mathrm{N}$ junction. The laser/photodiode, patented by this laboratory is defined in detail in the 2016 patent, published by the United States Patent and Trademark Office (USPTO). The references that include the in depth description of the new inventive art are numbered. ${ }^{2,3,8}$

\section{Future studies: here is where the market comes into play}

\section{In the epilepsy market: medications}

These BRODERICK PROBE® nanobiosensors currently have a global presence in the Epilepsy market for medicines because these nanobiosensors see inside the brain and these nanobiosensors have already contributed to advancement in this field.

These nanobiosensors have acceptance worldwide, in peer reviewed publications for the discovery of

a. neurochemically subtyping mesial and neocortical epilepsy in resected tissue in situ and en bloc.

b. peptides are biomarkers for long term neurodegeneration in the epilepsy patient.

c. dopamine and serotonin are biomarkers for early neurodegeneration In the brain of the epilepsy patient.

d. the presence of ascorbic acid as biomarker in the neocortical temporal lobe epilepsy brain of the epilepsy patient.

e. the presence of norepinephrine as biomarker in the mesial temporal lobe epilepsy brain of the epilepsy patient.

The Broderick team, in concert with NYU neurosurgeons and epileptologists, 22 publications, including 5 peer reviewed manuscripts and 17 major papers presented at Epilepsy Conferences globally. These conference sites included major surgical societies internationally.

The global epilepsy market is estimated to reach $\$ 4.5$ billion by 2019 . The epilepsy therapeutics market value in the eight major countries, that is, the US, Canada, France, Germany, Italy, Spain, the UK and Japan. The market will increase from \$3.4 billion in 2012 to $\$ 4.5$ billion by 2019 , at a modest Compound Annual Growth Rate (CAGR) of $3.9 \%$, says a new report from business intelligence provider GBI Research. According to the report, the US will grow at a higher CAGR of $4.8 \%$, climbing from $\$ 1.9$ billion in 2012 to $\$ 2.6$ billion by 2019. Meanwhile, the five European countries and Canada will achieve a combined, smaller CAGR of $3.1 \%$ during the forecast period. GBI Research attributes the anticipated market expansion to new Anti-Epileptic Drugs (AEDs) that have been approved during the last five years. However, further growth will be limited by the recent patent expiration of key AEDs, such as Keppra (levetiracetam) and Lamictal (lamotrigine). It may be true, as Global Intelligence has noted by Vullapalli, that the market has been dominated by GABA (gamma aminobutyric acid) and ion channel blockers. It is also true that "Second-generation AEDs, including levetiracetam, zonisamide and Vimpat (lacosamide), have signaled a shift in this trend in the last decade, with improved tolerability and safety through some discoveries". But the market is beset with the production of 'me too' drugs. Therefore, Vullapalli of GBI mentioned an urgent need for new mechanisms of action. Here is where BRODERICK PROBE ${ }^{\circledR}$ nanobiosensors will look inside the brain for the answers.

\section{In the epilepsy market: neurosurgery}

These BRODERICK PROBE ${ }^{\circledR}$ nanobiosensors currently have a global presence in the Neurosurgery market for Epilepsy because these nanobiosensors see inside the brain and these nanobiosensors have already contributed to advancement in this field.

The BRODERICK PROBE ${ }^{\circledR}$ series of nanobiosensors have already met with success in the epilepsy patient during resected tissue surgery in NYU Tisch Hospital under Institutional Review Board (IRB) Approval. These nanobiosensors have contributed to the epilepsy suegery market with the advancement of several discoveries with peer review and published approval, such as a surgical innovations gained from the success of these series of nanobiosensors in epilepsy patients during surgery, as listed below:

a. Miniature size allows precision

b. Less chance of infection postoperatively

c. Smaller margin of error

d. Faster recovery

e. Shortens hospital stays

f. Economically better for patient

g. More cost effective for health care system

h. Better quality of life for patient

i. Delays progression of disease

j. Lessens medications needed postoperatively

k. Also non invasive as well as minimally invasive

Epilepsy ranks fourth in most common neurology brain disorders. Alzheimer's, Parkinson's with Dementia, Dementia and acute ischemic stroke rank before epilepsy. But, epilepsy is a debilitating brain condition that can be fixed. In fact, surgery for resected tissue with corticography, has met with success in $95 \%$ of the patients presenting with epilepsy who are refractory to medications. In the United States alone, there are about more than 150,000 newly diagnosed cases every year. According to the World Health Organization, it is estimated that there are over 50 million people with Epilepsy. The remaining 30\%, or 15 million globally, are refractory to all forms of drug therapy and, therefore, these patients would be candidates for surgical treatment. From 1990 through 2008, there have been about 120,000 epilepsy surgeries performed in the United States alone. These data make the BRODERICK PROBE® series of nanobiosensors, a highly 
sought after imaging device for such intraoperative brain surgeries. It is the only medical device, globally, that enables live imaging of neurotransmitter profiles in the epilepsy brain on line and in real time. It is the only sensing device that performs this unique live imaging; it has brought not only the concept but the empirical answer to debilitated epilepsy patients.

The market for Stereotactic surgical devices gives the best insight into the global, market for epilepsy and Parkinson's diseases. The epilepsy disease segment held a lucrative market share in 2014 majorly on account of the effectiveness of the stereotactic surgery to provide safe treatment through smaller lesions near the critical structures of the head such as the brain stem. Trends to 2019 research report says global surgical imaging market was valued at an estimated $\$ 933.6$ million in 2014 and is expected to grow at a CAGR of $6.0 \%$ from 2014 to 2019. North America accounted for the largest share of the global surgical imaging market followed by Europe and Asia valued at $\$ 378.6$ million in 2014 .

\section{Summary}

Given the caveat that many surgeries and pharmaceuticals have helped to alleviate the miseries in each world of each epilepsy patient, it is humbly undeniable that the innovative discoveries discussed herein have contributed to the field of epilepsy both pre-clinically and clinically. Through the years, this laboratory has hopefully brought the epilepsy patient to a better quality of life with the BRODERICK PROBE $®$ carbon based series of nanobiosensors. The journey has led us to quantum mechanics in order to image new particles that may be toxic to the epilepsy patient and we did so by providing devices that can now image tiny packets of energized particles with the BRODERICK PROBE® protein neuroprobe. Finally, semiconductors, not only with quantum mechanics at the PN junction but with carbon fullerenes, brings this unique nanosensing/brain technology to utter heights of success for epilepsy patient pharmacotherapeutics and surgical advances. Thus, after several years of empirical discoveries for the epilepsy patient, we still have miles to go before we sleep.

The future of these nanobiosensors holds promise for the epilepsy patient with this discovery of the marriage between epilepsy and sensors. The journey from in Vivo Electrochemistry to Neuromolecular Imaging (NMI) to Voltaic Photonics with carbon and protein neuroprobes, minimally invasive and non-invasive appears poised to bode well for personalized medicine in an ever changing health care market. Toward the promise, a dialogue between the Singh Center of Nanotechnology at the University of Pennsylvania(U Penn) and CUNY's Broderick Nanotechnology is already initiated. Moreover, the founding of Eazysense Nanotechnology Inc. a company in collaboration with CUNY's Technology Commercialization Office, vested by Indian Angel Network ${ }^{\circledR}$ et al., can and will escalate the promise through process in both patient and veterinary medicine while the lab continues to further horizons manifold.

We have brought the world of sensors to the world of epilepsy and the author's final words are suitably "Sensors for Epilepsy Make Sense!"

\section{Acknowledgements}

The author wishes to thank the Broderick Brain Foundation, the F.M. Kirby Foundation, the Center for Advanced Technology, CUNY, and the MacKenzie Foundation, each for partial support of our laboratory and students during these studies. It is important to note that since this body of work, the development and pioneering of Neuromolecular Imaging and the BRODERICK PROBE ${ }^{\circledR}$ has taken place diligently for many years. Other grants including the National Institute of Health, National Institute on Drug Abuse, The Lowenstein Foundation, the FACES and PACE Foundations for Epilepsy, The Comprehensive Epilepsy Center, surgeon, Drs. Doyle, Kuzniecky, Devinsky and Pacia, NYU Anesthesiology and Neurology Departments, Drs. Becker (Haile and Li)and Chair, Dr. Kolodny as well as Dr. Piercey, Upjohn Pharmacia Company deserve honorable mention. Finally, we give special thanks to Eazysense Nanotechnologies Inc., its Board of Directors and CUNY TCO. Eazysense Nanotechnologies Inc. provides the company through which BRODERICK PROBE ${ }^{\circledR}$ nanobiosensors, vested by Indian Angel Network ${ }^{\circledR}$ et al., enables the conduit to market the nanobiosensors to treat animals and patients worldwide. The logo is shown in Figure 5.

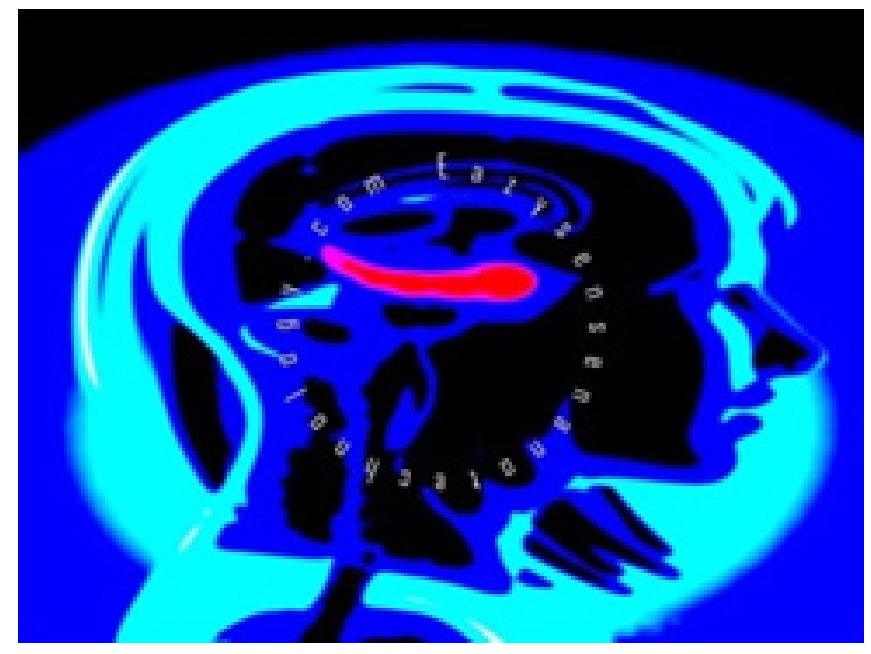

Figure 5 Logo for Eazysense Nanotechnologies Inc. Website is eazysensenanotechnology.com.

\section{Conflict of interests}

The authors declare there is no conflict of interest

\section{References}

1. Broderick PA. Real time signal processing at the neuromolecular level in the epilepsy and Parkinson's brain. IEEE Signal Processing in Medicine and Biology Symposium, Technical Program, Saturday, December 1, 2012 at the Grove School of Engineering, CCNY, in conjunction with the NYU Polytechnic Institute, New York.

2. Broderick PA. Noninvasive Electroactive Photonic Protein Sensor with Polymer Photovoltaic Optics for Memory Transduction using Organic and Inorganic Elements as Platforms. US Provisional Patent. 2015.

3. Broderick PA. Noninvasive Electroactive Photonic Protein Sensor with Polymer Photovoltaic Optics for Memory Transduction using Organic and Inorganic Elements as Platforms. US Non-Provisional Patent. 2016.

4. Broderick PA, Pacia SV. Identification, diagnosis, and treatment of neuropathologies, neurotoxicities, tumors, and brain and spinal cord injuries using electrodes with microvoltammetry. US Patent. 2012.

5. Broderick PA. Characterizing stearate probes in vitro for the electrochemical detection of dopamine and serotonin. Brain Res. 1989;495(1):115-121. 
6. Broderick PA. Microelectrodes and their use in cathodic electrochemical current arrangement with telemetric application. US Patent. 1995.

7. Broderick PA. Microelectrodes and their use in an electrochemical arrangement with telemetric application. US Patent. 1989.

8. Broderick PA. Noninvasive Photonic Sensor with Polymer Memory Transduction using Organic and Inorganic Elements as Platforms. USPTO Provisional Patent. 2015.

9. Broderick PA, Pacia SV. Identification, diagnosis, and treatment of neuropathologies, neurotoxicities, tumors, and brain and spinal cord injuries using microelectrodes with microvoltammetry. USPTO. 2006.

10. Broderick PA, Pacia SV. Identification, diagnosis, and treatment of neuropathologies, neurotoxicities, tumors, and brain and spinal cord injuries using microelectrodes with microvoltammetry. USPTO USSN. 2011.

11. Broderick PA. Distinguishing in vitro electrochemical signatures for norepinephrine and dopamine. Neurosci Lett. 1988;95(1-3):275-80.

12. Broderick PA. Cathodic Electrochemical Current Arrangement with Telemetric Application. US Patent. 1989.
13. Broderick PA. Studies of oxidative stress mechanism using a morphine/ ascorbate animal model and novel N-stearoyl cerebroside and laurate sensors. J Neural Transm. 2008;115(1):7-17.

14. Broderick PA, Pacia SV, Doyle WK, et al. Monoamine neurotransmitters in resected hippocampal subparcellations from neocortical and mesial temporal lobe epilepsy patients: in situ microvoltammetric studies. Brain Res. 2000;878(1-2):48-63.

15. Broderick PA, Ho H, Wat K, et al. Laurate biosensors image brain neurotransmitters in vivo: Can an antihypertensive medication alter psychostimulant behavior? Sensors. 2008;8(7):4033-4061.

16. Broderick PA, Pacia SV. Imaging white matter signals in epilepsy patients: A unique sensor technology. In: Broderick PA, Rahni DN, Kolodny EH, editors Bioimaging in Neurodegeneration, New Jersey, Springer, Humana Press Inc; 2005. p. 199-206.

17. Broderick PA. In vivo voltammetric studies on release mechanisms for cocaine with gammabutyrolactone. Pharmacol Biochem Behav. 1991;40(4):969-975. 